\title{
Stability control method of hybrid impulsive system based on M-Matrix
}

\author{
Yunxu Tong", Guihua Li, \\ College of Mathematics and Physics, Nanyang Institute of Technology, \\ Nanyang, 473004 \\ China
}

Received: June 14, 2021. Revised: December 19, 2021. Accepted: January 4, 2022. Published: January 5, 2022.

\begin{abstract}
Aiming at the problems of poor control effect and poor stability of the mixed pulse system with the traditional method, this paper introduces the M-matrix to establish the pulse delay differential indefinite formula and realize stability control of the mixed pulse system. The synchronization problem of mixed-pulse systems in complex networks is analyzed using $M$ matrix. The local coupling strength of the impulsive system is controlled according to the adaptive method. A class of Multi-Lyapunov functions is constructed for stability control of hybrid impulsive systems. The proposed method is proved to have better control effect through experiments.
\end{abstract}

Keywords-M-matrix, hybrid impulsive system, stability, control method.

\section{INTRODUCTION}

$\mathrm{W}$ ITH characteristics of fast response, good robustness and anti-jamming ability, impulse control has been widely used in the process of dynamic system control with parameter random perturbation [1], [2]. Hybrid impulsive system is a complex system consisting of discrete event system and continuous variable system, as well as a hybrid system with impulsive phenomena. Hybrid systems are widely used in hybrid automata control, aircraft control, automatic train control, flexible manufacturing system, networked control system and other practical issues [3]. In real life, there are many actual artificial or natural systems whose states change continuously in certain time intervals, and for some reason, their states will be suddenly changed at some time. Since the change time is often very short, the sudden change or jump process can be regarded as happening in a moment, so they are also called impulsive phenomena. This phenomenon can not be described by traditional continuous system or discrete system, so impulsive systems have been proposed to describe such dynamic control systems with impulsive phenomena. Impulsive systems can maintain and change any interesting or variable quantity in machine, mechanism or other equipment in the desired way. An important application of impulse control in control theory is chaotic system. Especially in the control of memristor chaotic systems, impulse control theory has important applications.

A stability control method for hybrid impulsive system based on stochastic perturbation neural network was proposed [4]. By studying the global exponential stability of a class of cellular neural networks with time-varying delays and parameter stochastic perturbations under impulse control, the global exponential stability problem was solved. The stability theory and discrete Halanay inequality technique were used to provide sufficient conditions for the global exponential stability of cellular neural networks with time-varying delays under hybrid impulsive control with and without parameter perturbations. Finally, numerical simulation were conducted to illustrate the results. This method can obtain the stability control coefficient of the pulse control system, and makes control process simpler, but the control effect is not satisfactory due to external interference. A stability control method for hybrid impulsive system based on predictive control was proposed [5]. Firstly, the hybrid impulse control was transformed into the system stability issue by using the consistency theory. Then, the predictive model was constructed, the sufficient conditions for the hybrid impulse stability control were deduced by using the impulse control protocol and Schur stability theorem, and the stability control of the hybrid impulsive system was realized. This method can effectively control the system, but the control process is more complicated and the control time is longer. A compatibility-based stability control method for hybrid impulsive system was proposed, and an impulsive $\mathrm{H} \infty$ control strategy was constructed for hybrid impulsive system with external perturbations [6]. On the premise that the system had a certain stability performance, impulse control was applied to slow state variables to avoid the destruction of system compatibility and to ensure that the impulse controlled system had a certain $\mathrm{H}_{\infty}$ performance. In order to reduce the conservativeness of the results, a time-dependent Lyapunov 
function with singular impulses was constructed by using the hybrid structure characteristics of closed-loop systems. The stability control of hybrid impulsive system was realized by combining convex combination technique and linear matrix inequality (LMIs) method. This method is only applicable to the closed-loop system, and the control effect object is relatively single. Reference [7] presented a stability control method for hybrid impulsive system based on CC-PT. Unlike traditional pulse sequence (PT) control technology, CC-PT control technology obtained two groups of high and low power control pulses with the same frequency and duty cycle change by comparing the sampling capacitive current with the preset high and low reference current. Taking the Buck converter of CC-PT control as an example, we described the working principle and analyzed its control characteristics, finding that this method has a shorter control time, but a less stable control effect.

In order to control the stability of hybrid impulsive system quickly and accurately, a stability control method of hybrid impulsive system based on M-matrix is proposed. An adaptive method is used to analyze the synchronization problem of mixed impulsive systems with Markov switching and adaptive local coupling strength. The M-matrix method is used to convert the system stability control problem into a mathematical problem. The effectiveness of the method is verified by simulation experiments.

\section{STABILITY CONTROL METHOD OF HYBRID IMPULSIVE SYSTEM}

The stability control flowchart is shown in Fig. 1.

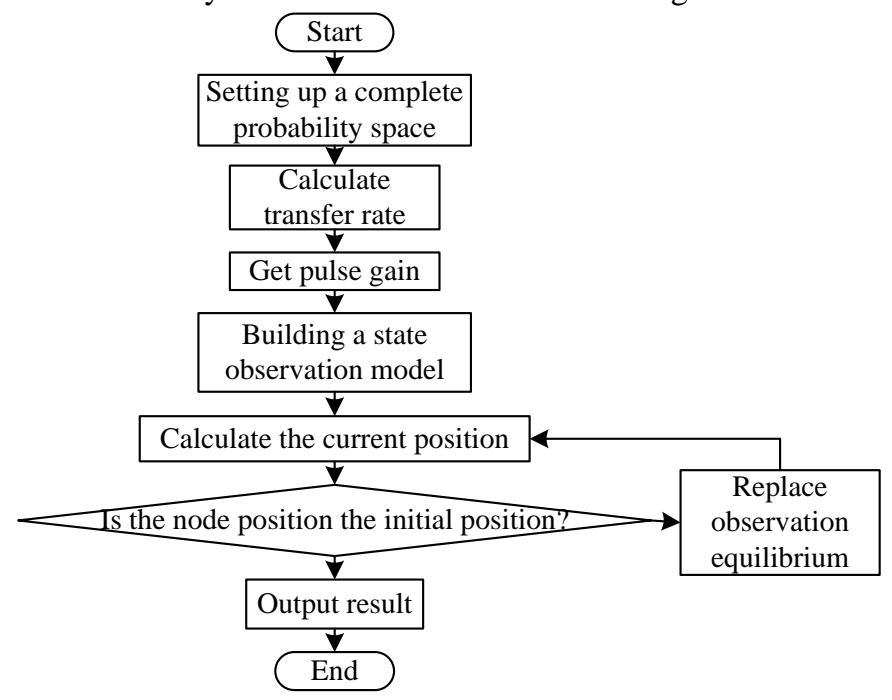

Fig. 1 Stability control process of hybrid pulse system

Analysis of Fig. 1 shows that, first, a complete probability space needs to be set to calculate the transfer rate. Then, the current pulse gain is obtained, and a state observation model is built to calculate the current position. At this time, it needs to judge whether the node position is the initial position, if yes, the result is output, the observation is replaced with the balance point anyway, and the node position is recalculated. Algorithm is designed according to this flowchart.

\section{A. Synchronization analysis of network under hybrid pulse}

This section focuses on the exponential mean square synchronization of complex networks with impulse and Markov switching. By using Lyapunov stability theory, impulse control principle and M-matrix method, the synchronization standard of the network is obtained. The specific process is as follows:

Supposing that $\{r(t), t \geq 0\}$ is a right continuous Markov chain in complete probability space $(\Omega, F, P)$. It takes a value in finite set $S=\{1,2, \cdots, k\}$ and its state transition matrix is $\left(\pi_{i j}\right)_{k \times k}$, which satisfies:

$P\{r(t+\Delta)=j \mid r(t)=i\}=\left\{\begin{array}{l}\pi_{i j} \Delta+0(\Delta), i \neq j \\ 1+\pi_{i i} \Delta+0(\Delta), i=j\end{array}\right.$

Where $\Delta>0, \pi_{i j} \geq 0$ represents the rate of transition from state $i$ to state $j$, and there is:

$\pi_{i i}=-\sum_{j \neq i}^{\prime} \pi_{i j}$

By considering a class of stochastic complex networks consisting of $N$ nodes, whose state equation is described as:

$d x_{i}(t)=\left\lfloor f\left(x_{i}(t), r(t)\right)+c(r(t)) \sum_{j=1}^{N} a_{i j}\left(r(t) x_{j}(t)\right) d t\right\rfloor+g\left(t, x_{i}(t), r(t) d w(t)\right)$

Where, $r(t), t>0$ is a continuous Markov process, $x_{i}(t)=\left[x_{i 1}(t), \cdots, x_{i n}(t)\right]^{T} \in R^{n}$ is the state of node $i$, $f\left(x_{i}(t), r(t)\right)=\left[f_{1} x_{i 1}(t), r(t), \cdots, f_{n} x_{i n}(t), r(t)\right]^{T} \quad$ is $\quad$ a continuous vector function, and the noise interference intensity $g\left(t, x_{i}(t)\right): R \times R^{n} \times S \rightarrow R^{n \times m}$ is a Borel measurable matrix function, $w(t)$ is a $m$-dimensional Brownian motion, $\Gamma(r(t))=\operatorname{diag}\left(\gamma_{1, r(t)}, \cdots, \gamma_{n, r(t)}\right)$ is an internal coupling matrix, and $c(r(t))$ is a coupling strength.

$A(r(t))=\left(a_{i j}(r(t))\right) \in R^{N \times N} \quad$ is the external coupling matrix, and its element $a_{i j}(r(t))$ is set as follow: if node $i$ and node $j$ have information exchange, then $a_{i j}(r(t))=a_{j i}(r(t))>0$, otherwise $a_{i j}(r(t))=0$, and its diagonal element is set as:

$$
a_{i i}(r(t))=-\sum_{j=1}^{N} a_{i j}(r(t))=-\sum_{j=1}^{N} a_{j i}(r(t))
$$

For networks with impulsive interference and impulses only occurring on some nodes [8], the equation of state can be described as:

$$
\begin{aligned}
& d x_{i}(t)=\left\lfloor f x_{i}(t), r(t)+c(r(t)) \sum_{j=1}^{N} a_{i j}(r(t)) \Gamma(r(t)) x_{j}(t)+u_{i}(t)\right\rfloor d t \\
& +g\left(t, x_{i}(t), r(t)\right) d w(t)
\end{aligned}
$$

Where, 
$u_{i}(t)=\sum_{k=1}^{\infty} b_{i k}\left(x_{i}\left(t_{k}\right)-s(t)\right) \delta\left(t-t_{k}^{i}\right)$

$b_{i k}$ is the pulse gain of node $i$ at time $t_{k}^{i}$ and $\delta(t)$ is the Dirac Delta function. $s(t)$ is a single isolated node, which is described as:

$d s(t)=f(s(t), r(t)) d t+g(t, s(t), r(t)) d w(t)$

For each subscript $i$, set $t_{k}^{i}$ satisfies $0 \leq t_{0}^{i}<t_{1}^{i}<\cdots<t_{k}^{i}$, and when $k \rightarrow+\infty, t_{k}^{i} \rightarrow+\infty$.

For all $i$ and $k, t_{k}^{i}$ is rearranged to obtain a new sequence [9], which is denoted as $t_{k}$, so that $t_{0}<t_{1}<\cdots<t_{k}$. Equivalent formula (6) can be rewritten as follows:

$\left\{\begin{array}{l}d x_{i}(t)=\left\lfloor f\left(x_{i}(t), r(t)\right)+c(r(t)) \sum_{j=1}^{N} a_{i j}(r(t)) \Gamma(r(t)) x_{j}(t) d t\right\rfloor+g\left(t, x_{i}(t), r(t)\right) d w(t) \\ \Delta x_{i}\left(t_{k}\right)=x_{i}\left(t_{k}^{+}\right)-x_{i}\left(t_{k}^{-}\right)=b_{i k}\left(x_{i}\left(t_{k}^{-}\right)-s(t)\right), t=t_{k}, k \in Z^{+}, i=1,2, \cdots, N\end{array}\right.$

When $t_{k}=t_{k}^{i}, b_{i k}=b_{i k^{\prime}}$, otherwise $b_{i k}=0$. For each $t_{k}$, there is at least one $t_{k}^{i}$ corresponding to it.

The main objective of this section is to enable the network to achieve exponential synchronization and to find sufficient conditions for synchronization:

If the synchronization error $e_{i}(t)=x_{i}(t)-s(t)(i=1,2, \cdots, N)$, the error system can be described as follows:

$\left\{\begin{array}{l}d e_{i}(t)=\left\lfloor f\left(e_{i}(t), r(t)\right)+c(r(t)) \sum_{j=1}^{N} a_{i j}(r(t)) \Gamma(r(t)) e_{j}(t) d t\right\rfloor+g\left(t, e_{i}(t), r(t)\right) d w(t) \\ \Delta e_{i}\left(t_{k}\right)=e_{i}\left(t_{k}^{+}\right)-e_{i}\left(t_{k}^{-}\right)=b_{i k}\left(e_{i}\left(t_{k}^{-}\right)-s(t)\right), t=t_{k}, k \in Z^{+}, i=1,2, \cdots, N\end{array}\right.$

Where,

$f\left(e_{i}(t), r(t)\right)=f\left(x_{i}(t), r(t)\right)-f(s(t), r(t)), g(t, e(t), r(t))=g\left(t, x_{i}(t), r(t)\right)-g(t, s(t), r(t))$

Considering stochastic differential equations with Markov switching, the equation of state is described as:

$d x(t)=f(t, r(t), x(t)) d t+g(t, r(t), x(t)) d w(t)$

If there are two constants $M_{0}>0, \eta>0$, there are:

$E\left\{\|x(t)\|^{2}\right\} \leq M_{0} e^{-\eta t}$

If all the initial values $x(0) \in R^{n}$ are valid, the system is said to be exponentially stable [10].

For a pulse sequence $\varsigma=\left\{t_{1}, t_{2}, \cdots\right\}$, if there exists a positive integer $N_{0}$ and a positive integer $T_{a}$, such that:

$\frac{T-t}{T_{a}}-N_{0} \leq N_{\varsigma}(T, t) \leq \frac{T-t}{T_{a}}+N_{0}, \forall T \geq t \geq 0$

$T_{a}$ is the average pulse time of the pulse sequence $\varsigma$, and $N_{\varsigma}$ is the total number of pulses in the center of the pulse sequence on the interval $(t, T)$.

Asynchronous pulses contain impulse interference, which has adverse effects on network synchronization [11]. The synchronization mechanism of complex networks with asynchronous pulses and Markov handover is given below.

The non-negative Lyapunov function is expressed as follow:

$V(t, r(t) ; e(t))=\frac{q_{r(t)}}{2} \sum_{i=1}^{N} e_{i}^{T}(t) e_{i}(t), r(t) \in S$

For arbitrary pulse interval $\left[t_{k}, t_{k+1}\right]$, considering that $r(t)$ is a finite right continuous piecewise function in $\left[t_{k}, t_{k+1}\right]$. For the sake of generality, it is assumed that there are $l_{k}$ jumps on $\left[t_{k}, t_{k+1}\right]$, i.e. $t_{k} \leq t_{k, 1} \leq \cdots t_{k+1}$. This also means that $r(t)$ takes the unique value on the interval $t \in\left\lfloor t_{k, s}, t_{k, s+1}\right\rfloor$.

Let $r(t)=\sigma$, then Lyapunov function can be rewritten as:

$V(t, \sigma, e(t))=\frac{q_{\sigma}}{2} \sum_{i=1}^{N} e_{i}^{T}(t) e_{i}(t), t \in\left[t_{k, s}, t_{k, s+1}\right]$

For each $\sigma$, operator $L V(t, \sigma, e(t))$ along the error system can be calculated, and there are:

$L V(t, \sigma, e(t))=q_{\sigma} \sum_{i=1}^{N} e_{i}^{T}(t)\left[f e_{i}(t), \sigma\right]+c_{\sigma} a_{i j}^{\sigma} \Gamma e_{j}(t)$

Considering that the coupling matrix $A_{\sigma}$ is symmetric, there are:

$e_{j}^{-T}(t) A_{\sigma} e_{j}(t) \leq \lambda_{\max }\left(A_{\sigma}\right) e_{i}^{T}(t)=0$

According to the above theorem conditions, it can be concluded that:

$L V(t, \sigma, e(t)) \leq-\frac{\sigma}{2} \sum_{i=1}^{N} e_{i}^{T}(t) e_{j}(t) \leq-p V(t, \sigma, e(t))$

Where,

$q=\max _{i \in S} q_{i}, p=\frac{\alpha}{q}$

Let $W(t, r(t), e(t))=e^{p t} V(t, r(t), e(t))$, then:

$L W(t, r(t), e(t))=p e^{p t} v(t, r(t), e(t))+e^{p t} L V(t, \sigma, e(t))$

Based on formula (19), we can see that:

$E W(t, r(t), e(t))=E W\left[t_{k}, t_{k+1}\right]+E \int_{t k}^{t} L W(t, r(t), e(t)) d s$

Thus,

$E V(t, r(t), e(t)) \leq E V\left(t_{k}, r\left(t_{k}\right), e\left(t_{k}\right)\right) e^{-p\left(t-t_{k}\right)}$

On the other hand, the structure of $V(t, r(t), e(t))$ shows that:

$V(t, r(t), e(t))=\frac{q_{r\left(t_{k}\right)}}{2} \sum_{i=1}^{N} e_{i}^{T}(t) e_{j}(t) \leq-p V(t, \sigma, e(t))$

Where,

$p=\max \left\{\frac{q}{q}\left(1+b_{i k}\right)^{2}, i=1,2, \cdots, N, K \in Z^{+}\right\}$

For any $K \in Z^{+}$, when $t \in\left(t_{k}, t_{k+1}\right)$, according to the conditions in formula (22) and related theorems, there are:

$E V(t, T(s), e(t)) \leq \rho E V\left(t_{k}^{-}, r\left(t_{k}\right), e\left(t_{k}\right)\right) e^{-p\left(t-t_{k}\right)}$ 
Thus,

$E e_{i}^{T}(t) e_{i}(t) \leq \frac{1}{q} E V(t, \sigma, e(t))$

Synchronization pulse, also known as pulse control, can promote network synchronization [12]. In order to obtain the synchronization mechanism of the network under synchronous pulses, assuming that network $b_{i k^{\prime}}=b_{k}, t_{k}^{i}=t_{k}$ holds for all $i$, that is to say, the gain of pulse is the same and the time of pulse occurrence is the same. The synchronization mechanism of the network under synchronous pulse can be expressed as follows.

Choosing Lyapunov function which is the same as theorem 3.1 and calculating operator $L V(t, \sigma, e(t))$ and related theorems, we have:

$$
L V(t, \sigma, e(t)) \leq q_{\sigma} \sum_{i=1}^{N} e_{i}^{T}(t) e_{j}(t)
$$

The above process mainly studies the synchronization of stochastic complex networks with impulse and Markov switching topologies. The network considered not only has impulse and noise perturbations, but also has $k$ different topologies, which are switched randomly according to Markov chains. Based on Lyapunov stability theory and M-matrix method, synchronization criteria for such complex networks are obtained.

\section{B. Construction of stability controller for hybrid impulsive} system

In the study of the stability of hybrid impulsive system, the asymptotic stability is the focus. The asymptotic stability of dynamic systems means that when the time tends to be infinite, the trajectory of the system converges to the equilibrium point of the system. However, in many practical problems, the state trajectory of the system is often required to converge to the equilibrium point of the system in a finite time. In this way, the concept of finite-time stability is proposed, which requires that the system to be not only asymptotically stable, but also converge to the equilibrium point of the system in a finite time. Obviously, the concept of finite-time stability overwhelms the concept of asymptotic stability.

Suppose there is a hybrid dynamic system, where $\mathrm{y}$ is the measurable output variable. Assuming that $f_{i k}\left(x_{[k]}\right)$ satisfies the global Lipschitz condition, and taking the Lipschitz constant as $l_{i}, k=1,2, \cdots, n$, we have:

$x\left(t_{j, r}^{+}\right)=G_{j} x\left(t_{j, r-1}^{+}\right)+F_{j} u\left(t_{j, r}^{+}\right)$

If,

$i=q\left(t^{-}\right) \neq q\left(t^{+}\right)=j, i, j=1,2, \cdots, m, k=1,2, \cdots, n$

Where $G_{j}=\operatorname{diag}\left\{g_{j 1}, g_{j 2}, \cdots, g_{j n}\right\}$ is a diagonal matrix with known appropriate dimensions, $t^{+}=t_{j, r}^{+}, \quad$ and $i, j=1,2, \cdots, m, k=1,2, \cdots, n$.

Since the hybrid impulsive system consists of two parts, the corresponding hybrid observer also consists of two parts, namely, the position observer of the discrete event subsystem and the state observer of the continuous variable subsystem.

$\left\{\begin{array}{l}q\left(t_{k+1}\right) \in \varphi_{0}\left(q\left(t_{k}\right), \psi\left(t_{k+1}\right)\right) \\ \psi_{0}\left(t_{k+1}\right)=q\left(t_{k}\right)\end{array}\right.$
$\left\{\begin{array}{c}\hat{x}_{1}=\hat{x}_{2}+L_{i} a_{i 1}\left\langle e_{1}\right\rangle^{a_{i l}}+f_{i 1}\left(\hat{x}_{[1]}\right) \\ \hat{x}_{2}=\hat{x}_{3}+L_{i}^{2} a 2\left\langle e_{1}\right\rangle^{a_{i l}}+f_{i 2}\left(\hat{x}_{[2]}\right) \\ \vdots \\ \hat{x}_{k}=\hat{x}_{k+1}+L_{i}^{k} a_{i k}\left\langle e_{1}\right\rangle^{a_{i l}}+f_{i l}\left(\hat{x}_{[k]}\right) \\ \vdots \\ L_{i}=l_{i} \lambda_{i} L_{i}\end{array}\right.$

Where formula (29) represents a position observer and formula (30) represents a state observer of the system. The position set $Q_{0} \in 2^{Q}$, the input set $\sum_{0}=\psi$ of the position observer and the output set of the position observer $\psi_{0=} Q_{0}$. $q\left(t_{k}\right)$ represents the position estimation of the system at time $\mathrm{t}$, that is, the input of the position observer is basically the output of the FSM, the output of the position observer is basically the position estimation $\tilde{q}\left(t_{k}\right)$ of the current position $q\left(t_{k}\right)$ of the system, and $\hat{x}$ is the estimation of the state $x$, where $L_{i}$ is the observation gain. The gain of the controller is a dynamic variable, satisfying $L(0) \geq 1$ and $\lambda_{i}>0$, while $e_{1}=x_{1}-\hat{x}_{1}$, the corresponding matrix is:

$\Omega_{i} \square\left[\begin{array}{ccc}-a_{i l} & 1 & 0 \\ \vdots & \vdots & \vdots \\ -a_{i, n-1} & 1 & 0 \\ -a_{i n} & 1 & 0\end{array}\right\rfloor$

If the dynamic system of discrete event and its position observer satisfy the following conditions;

(1) The intersection of the position set $Q$ and the observer's position set $Q_{0}$ is not empty;

(2) The intersection of each basic ring $Q_{c}^{i}$ and position set $Q$ is not empty;

(3) The intersection of the position set $Q$ and the observer's position set $Q_{0}$ is $\varphi_{0}$ and it is invariant.

The position observer in the hybrid observer can determine the current position of the system in a finite area, while the state observer can only start working after the position observer accurately determines the current position of the system.

Design of position observer: Since the initial position of the FSM is uncertain, the current position of the system state is estimated to be $q\left(t_{0}\right)=Q$, that is, the position estimation $q\left(t_{0}\right)$ contains all the positions of the FSM. When the first output event $\psi\left(t_{1}\right)$ of the FSM occurs, the observer receives the first input event. According to $\tilde{q}\left(t_{1}\right) \in \varphi_{0}\left(\tilde{q}\left(t_{0}\right), \psi\left(t_{1}\right)\right)$, the current position estimation $\tilde{q}\left(t_{1}\right)$ of the system state can be obtained. According to the finite state automaton, the position 
of the initial position $q\left(t_{0}\right)$ is estimated to be $\tilde{q}\left(t_{0}\right)=\{1,2,3,4,5\}$. If the first output event of the FSM is $\psi\left(t_{1}\right)=\alpha$, then $\tilde{q}\left(t_{1}\right)=\{2,4\} \quad ; \quad$ If $\psi\left(t_{1}\right)=\lambda$, then $\tilde{q}\left(t_{1}\right)=\{3,5\}$; If $\psi\left(t_{1}\right)=\mu$ continues according to this process, the current position of the system will be accurately determined after a finite step, that is, $\tilde{q}\left(t_{k}\right)$ contains only one position point $q\left(t_{k}\right)$.

The design of state observer: according to the characteristics of the hybrid observer, the state observer can only start to work after the position observer accurately determines the current position of the system.

It is assumed that the corresponding observation error system can be obtained from the above process:

$e\left(t_{j, r}^{+}\right)=G_{j} e\left(t_{j, r-1}^{+}\right)$

Where,

$e_{k}=x_{k}-\hat{x}_{k}, \hat{f}_{i k}=f_{i k}\left(x_{[k]}\right)-f_{i k}\left(\hat{x}_{[k]}\right)$

In the form of the above process, a hybrid dynamic observer is designed. The detailed research process is given below.

According to the above lemma, we first set the multi-Lyapunov function $V_{i}(\tilde{\varepsilon})=\tilde{\varepsilon} P_{i} \tilde{\varepsilon}$ and $P_{i}$ is positive definite symmetric matrix. Obviously, if $\tilde{\varepsilon}$ is finite-time stable, we can conclude that $\varepsilon$ is also finite-time stable. Through the relevant theorems in prior knowledge, we can see that:

$V_{i}(\varepsilon)=\tilde{\varepsilon} P_{i} \frac{x_{k}-\widehat{x}_{k}, \widehat{f}_{i k}}{f_{i k}\left(x_{[k]}\right)-f_{i k}\left(\widehat{x}_{[k]}\right)} P_{i}^{k, r}$

Where, $P_{i}^{k, r}$ represents the element $r$ of $P_{i}$ in row $k$.

From the above discussion, the following conclusions can be drawn:

(1) The discrete event subsystem is observable;

(2) Before the position observer determines the current position of the system, the control $u=0$ and $\tilde{u}=0$ are added to the hybrid system, and the Jacobian matrix $\frac{\partial\left(A x+F_{i}(x)\right)}{\partial x^{T}}$ of the system becomes the Hurwit matrix.

(3) After the position observer determines the current position of the system.

A number of researches on guaranteeing finite-time stability of hybrid impulsive system have been conducted, but there is a lack of researches on hybrid impulsive system, especially the finite-time observer of hybrid impulsive system. This section designs a class of impulsive hybrid observers for hybrid impulsive system. The designed observer is a finite-time observer, which can realize the stability control of hybrid impulsive system based on M-matrix. for hybrid impulsive system based on M-matrix, simulation experiments are carried out using a PC with Intel Core i7-6700HQ processor, 16GB memory, 1TB disk and Windows 10 operating system. The proposed method, the stability control method of hybrid impulsive system based on stochastic perturbation neural network, the stability control method of hybrid impulsive system based on predictive control, the stability control method of hybrid impulsive system based on compatibility and the stability control method of hybrid impulsive system based on CC-PT are comparatively analyzed in terms of control performance.

\section{A. Control time under different methods}

The control time (min) of different control methods are comparatively analyzed, and the comparison results are shown in Fig. 2.

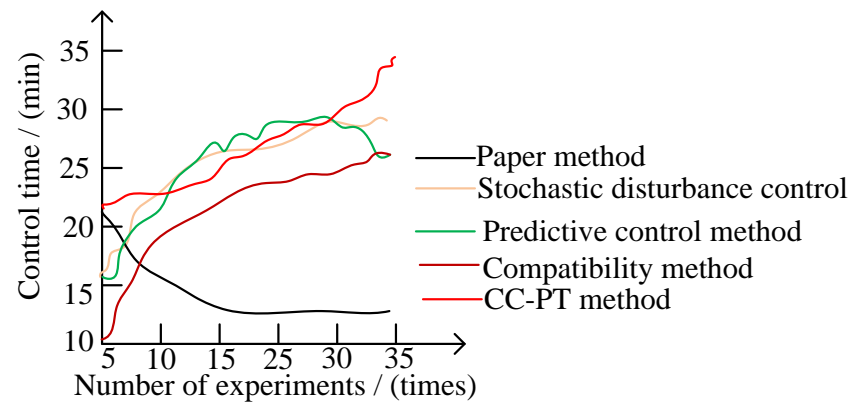

Fig. 2 Control time of different stability control methods

From Fig. 2, it can be seen that the control time of the proposed method is longer in the initial stage, begins to show a downward trend with the increase of the number of experiments, and then tends to be stable. The control time of the four methods for hybrid impulse system are ranked from short to long as the method based on stochastic perturbation neural network, predictive control method, compatibility control method and CC-PT control method, but all of them require significantly longer control time than the proposed method. The experimental data show that the proposed method can effectively reduce the control time and improve the efficiency of the whole system.

\section{B. Comparison of system stability control errors}

The proposed method, the stability control method of hybrid impulsive system based on stochastic perturbation neural network proposed in [4], the stability control method of hybrid impulsive system based on predictive control proposed in [5], the stability control method of hybrid impulsive system based on compatibility proposed in [6], and the stability control method of hybrid impulsive system based on CC-PT proposed in [7] are comparatively analyzed in terms of the control error, as shown in Table I.

\section{EXPERIMENTAL SIMULATION}

In order to prove the validity of the stability control method

Table. I Comparison results of control errors for different control methods

Sample size /

Control error / (\%) 
INTERNATIONAL JOURNAL OF CIRCUITS, SYSTEMS AND SIGNAL PROCESSING

\begin{tabular}{cccccc}
\cline { 2 - 5 } (unit) & $\begin{array}{c}\text { Proposed } \\
\text { method }\end{array}$ & $\begin{array}{c}\text { The method in } \\
{[4]}\end{array}$ & $\begin{array}{c}\text { The method in } \\
{[5]}\end{array}$ & $\begin{array}{c}\text { The method in } \\
{[6]}\end{array}$ & The method in [7] \\
\hline 1000 & 1.25 & 0.97 & 1.20 & 1.36 & 1.58 \\
2000 & 1.21 & 1.11 & 1.35 & 1.47 & 1.79 \\
3000 & 1.19 & 1.35 & 1.44 & 1.59 & 2.05 \\
4000 & 1.02 & 1.41 & 1.58 & 1.68 & 2.84 \\
5000 & 0.87 & 1.52 & 1.67 & 1.77 & 3.22 \\
Average error / & 1.108 & 1.272 & 1.448 & 1.574 & 2.296 \\
(\%) & & &
\end{tabular}

By analyzing the experimental data in Table I, when the number of samples is 1000 , the control error of the method in [4] is $0.97 \%$, that of the method in [5] is $1.20 \%$, that of the method in [6] is $1.36 \%$, that of the method in [7] is $1.58 \%$, and the that of the proposed method is $1.25 \%$. When the number of samples is 2000 , the control error of the method in [4] is $1.11 \%$, which of the method in [5] is $1.35 \%$, which of the method in [6] is $1.47 \%$, that of the method in [7] is $1.79 \%$, and that of the proposed method is $1.21 \%$. When the number of samples is 5000 , the control error of the method in [4] is $1.52 \%$, which of the method in [5] is $1.67 \%$, which of the method in [6] is $1.77 \%$, that of the method in [7] is $3.22 \%$, and that of the proposed method is $0.87 \%$. The control error of the proposed method is the lowest among the five methods, and the stability control method in [4] is second only to the proposed method. The control error of the other three methods is high, so they cannot meet the requirements of output stability control of hybrid pulse system. The experimental results show that the proposed method can effectively ensure the stable operation of the hybrid pulse system.

\section{A. Comparison of system control stability}

In order to verify the effectiveness of the proposed method, the control stability of four different methods are comparatively analyzed, and the results are shown in Fig. 3.

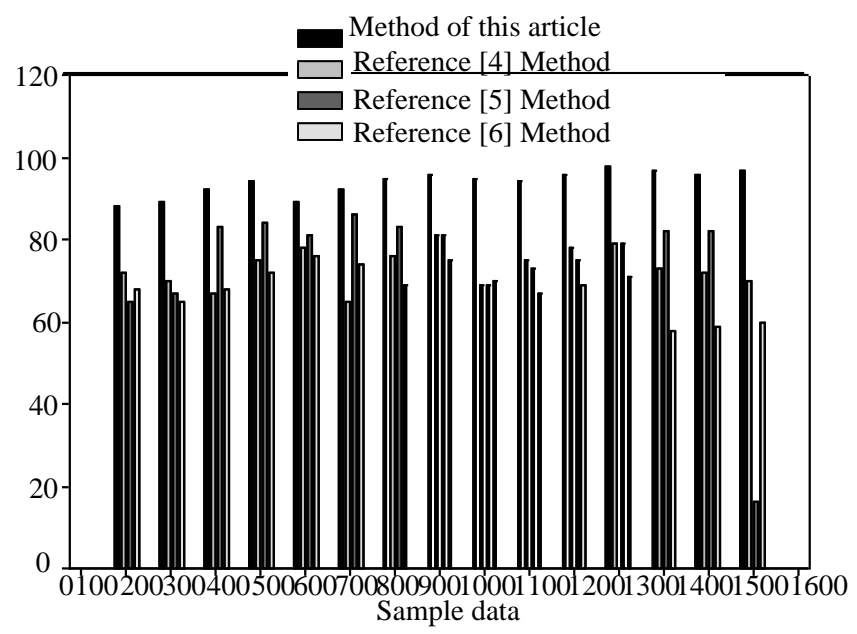

Fig. 3 Control stability of different methods

Fig. 3 shows that the stability of the control system under different sample numbers. Taking the sample data equal to 500 as an example, the stability of the method in [4] is $78 \%$, which of the method in [5] is $81 \%$, that of the method in [6] is $76 \%$, and that of the method in this paper is $89 \%$. The stability of the method in this paper is the highest. Taking the sample data equal to 1000 as an example, the stability of the method in [4] is $75 \%$, which of the method in [5] is 73\%, that of the method in [6] is $67 \%$, and that of the method in this paper is $94 \%$. The stability of the method in this paper is the highest. From the analysis of the overall results, the proposed method always has the highest stability.

\section{CONCLUSIONS}

In recent years, great achievements have been made in the study of the stability of impulsive systems. Synchronization, as an important research direction of complex networks connected with impulsive systems, has attracted the attention of many scholars, and relevant research results have been widely applied in many fields, such as biology, computer, economy and so on. Based on the traditional methods, the stability control of hybrid impulsive systems connected to complex networks is systematically studied in this paper, and the main results are summarized as follows:

(1) A complex network model with switching topology connected to impulsive systems is considered, and the global exponential synchronization of such networks is studied. The topological structure of this kind of network is changing with time. Compared with the previous complex network model with fixed topological structure, it is more general and more practical. By using Lyapunov stability theory and impulse control principle, some criteria for synchronization of such switched complex networks are obtained. These criteria depend on the network topology, pulse gain and delay. In addition, a new impulsive delay differential inequality is proposed, which is very effective in estimating the state of impulsive unstable networks.

(2) Hybrid dynamic system theory involves discrete events and continuous variables. The research on hybrid dynamic system is one of the hot topics in the current control field. The proposed method mainly focuses on a class of impulsive hybrid dynamic systems. The main work includes: 1) Impulsive hybrid controller is designed for hybrid impulsive system by using Back stepping method; 2) A hybrid high gain observer is designed for hybrid impulsive system, so it becomes a finite time observer; 3) For a class of hybrid impulsive system, a hybrid feedback controller based on hybrid observer is designed.

After decades of development, the research on hybrid impulse systems has achieved a lot of results, and new theories and methods have been increasingly emerging. However, due to the complexity of the hybrid impulse system and the lack of 
analysis tools, many valuable problems need to be solved. The specific issues are as follows:

(1) In this paper, the switching phenomenon and pulse phenomenon are considered to be time triggered, that is, the switching time and pulse time are assumed to be given. If the pulse time and switching time are related to the system state, they need to be designed in the process of stability analysis. How to design pulse time series or switching time series to ensure the correlation stability of the system is worthy of further research.

(2) In this paper, the systems we consider are special forms of random hybrid systems. For general random hybrid systems, because the existing theory is not perfect, this direction is also worthy of in-depth research, such as hybrid systems with time delay and general random hybrid systems.

In short, the research and application of the theory of mixed pulse systems are still in its infancy, and many meaningful problems need to be solved. It is believed that through the continuous efforts of non-mathematicians, the mixed pulse system will become a complete scientific system.

\section{References}

[1] Q.H. Hong, Y. Li, X.P. Wang, and Z.G. Zeng, "A versatile pulse control method to generate arbitrary multi-direction multi-butterfly chaotic attractors," IEEE Transactions on Computer-Aided Design of Integrated Circuits and Systems, pp. 1-1, 2018.

[2] J.T. Yu, H.P. Li, Q.Y. Nie, Z.Y. Zou, H.Q. Liu, C.Y. Bao, Y.X. Jie, and Z.X. Li, "Note: Stability control of intermediate frequencies of a three laser far-infrared polarimeter-interferometer system," Review of Scientific Instruments, vol. 87, no. 12, pp. 126102, 2016.

[3] C.S. Wu, J. Chen, C.F. Xu, and Z.Y. Liu, "Real-time adaptive control of a fuel cell/battery hybrid power system with guaranteed stability," IEEE Transactions on Control Systems Technology, pp. 1-12, 2016.

[4] Y.Q. Chen, "Impulsive control of randomly perturbed neural networks," Journal Applied Math, no. 40, pp. 16-26, 2017.

[5] G.L. Zhang, B.Y. Du, Y.J. Sun, and W.J. Tang, "Impulsive control for multi-robot formation with communication delay based on predictive control," Control and Decision, vol. 31, no. 8, pp. 1453-1460, 2016.

[6] X.M. Lu, Z. Ruan, and W.H. Chen, "Impulsive Hळ control of a class of linear singular time-delay systems," Journal of Guangxi University (Natural Science Edition), vol. 42, no. 6, pp. 2030-2039, 2017.

[7] Y.T. Zhao, X. Chen, H.Y. Chen, Y. Guan, and J. Zhu, "Research on active protection system of electric vehicle charging facility based on active hybrid pulse charging strategy," Automobile Technology, no. 08, pp. 27-31, 2019.

[8] W. Xu, K.J. Xu, L.P. Liang, and S.L. Yang, “Analysis and development of electromagnetic flowmeter pulse excitation system based on PWM control," Acta Metrologica Sinica, vol. 39, no. 4, pp. 525-530, 2018.
[9] A. Martin, E. Schiavi, Y. Eryaman, J. Lopez Herraiz, B. Gagoski, E. Adalsteinsson, L. Wald, and B. Guerin, "Parallel transmission pulse design with explicit control for the specific absorption rate in the presence of radiofrequency errors," Magnetic Resonance in Medicine, no. 75, pp. 2493-2504, 2015.

[10]B. Yang, B.J. Wei, Y.M. Wei, and J. Mao, "Hybrid switching control method for micro-satellite station keeping," Chinese Space Science and Technology, vol. 38, no. 04, pp. 20-26, 2018

[11]Z. Guo, S.Z. Yu, Z. Li, and J.C. Guo, "Research on application of hybrid energy storage management strategy based on fuzzy logic for ship medium-voltage DC power system," Journal of Power Supply, vol. 17, no. 3, pp. 172-181, 2019.

[12] W. Tan, B. Li, B. Zhou, and M. Fang, "Pinning adaptive impulsive hybrid synchronization of fractional order complex dynamic network," Journal of Hunan University of Science \& Technology (Natural Science Edition), vol. 34, no. 2, pp. 80-89, 2019.

[13] Y.T. Zhao, X. Chen, and H.Y. Chen, "Research on active protection system of electric vehicle charging facilities based on active hybrid pulse charging strategy," Automotive Technology, vol. 01, no. 08, pp. 27-31, 2019.

[14] R.X. Wei, T. Ni, X.L. Zhao, and X.Y. Zhang, "Collision avoidance stability of the cognitive unmanned aerial vehicle \& environment system," Control Theory \& Applications, vol. 36, no. 09, pp. 1453-1460, 2019.

[15]H.G. Wu, Y.J. Niu, and S.N. Hu, "Impulsive synchronization of unified chaotic system under white noise," Mathematical Practice and Understanding, vol. 49, no. 05, pp. 290-296, 2019.

[16] J.Y. Wei, J.S. Ren, and L. Chen, "Power control of morwpt system based on complex hybrid modulation," Power Electronics, vol. 53, no. 08, pp. 107-110, 2019.

[17] Y.T. Li, F.B. Chen, and D.F. Wang, "New lower bounds on eigenvalue of the Hadamard product of an M-matrix and its inverse," Journal of Sichuan University of Science \& Engineering, vol. 430, no. 4, pp. 1423-1431, 2009.

[18]F. Mirzaee, S. Alipour, and N. Samadyar, "Numerical solution based on hybrid of block-pulse and parabolic functions for solving a system of nonlinear stochastic It-Volterra integral equations of fractional order," Journal of Computational and Applied Mathematics, vol. 349, no. 21, pp. 157-171, 2019.

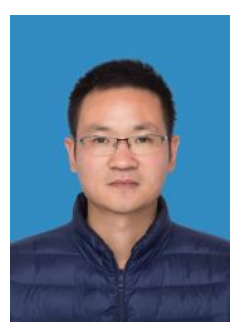

Yunxu Tong, male, born in June 1981, and He had got the B.S. in 2005, from Anyang Normal University, majoring in mathematics. Then he was awarded the Ph.D. in 2014,from Shaanxi Normal University, majoring in control theory. Now he is working in Nanyang Institute of Technology. And his 
research areas is finite-stability and filtering of impulsive systems. He has published 15 academic papers. Meanwhile, he has participated in five cooperative research projects.

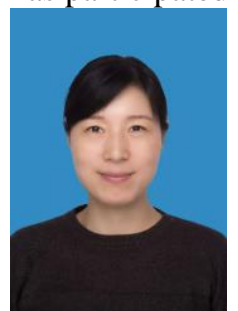

Guihua Li, female, born in September 1981, and she had got the B.S. in 2005, from Anyang Normal University, majoring in mathematics. Now she is working in Nanyang Institute of Technology. And her research areas is mathematics and applied mathematics. She has published 7 academic papers. Meanwhile, she has participated in three cooperative research projects.

\section{Contribution of individual authors to the creation of a scientific article}

Yunxu Tong and Guihua Li proposed a stability control method of hybrid impulsive system based on M-matrix. Yunxu Tong analyzed the synchronization problem of hybrid impulsive system with Markov switching complex network by M-matrix method. The synchronization problem of hybrid impulsive systems with Markov switching and adaptive local coupling strength is analyzed by adaptive method. Guihua Li constructs a mixed pulse observer and a mixed pulse feedback controller for mixed pulse systems. Under the combined action of the two, the closed-loop system is asymptotically stable. A class of multi Lyapunov functions is constructed and applied to the stability control of hybrid impulsive systems. Yunxu Tong and Guihua $\mathrm{Li}$ conducted experiments and recorded data. And write manuscripts. All authors read and approved the final manuscript.

\section{Creative Commons Attribution License 4.0 (Attribution 4.0 International, CC BY 4.0)}

This article is published under the terms of the Creative Commons Attribution License 4.0

https://creativecommons.org/licenses/by/4.0/deed.en_US 